\title{
SURGICAL REHABILITATION OF NASAL SEPTUM PERFORATION IN CHILDREN
}

\author{
Adnan Yunusov ${ }^{1,2}$, Nikolai Daykhes ${ }^{1,2}$, Hassan Diab ${ }^{1,2}$, Ekaterina Molodtsova ${ }^{1}$ \\ ${ }^{1}$ Federal State Budgetary Institution "Clinical Research Centre for Otorhinolaryngology to \\ the Federal Medico-Biological Agency of the Russian Federation”, Moscow, Russia \\ ${ }^{2}$ Pirogov Russian National Research Medical University
}

\begin{abstract}
INTRODUCTION: Technical difficulties and failures are also determined by the fact that it is extremely rare to close the perforation by simple mutual displacement and cross-linking of the flaps of mucoperichondrium.

The aim of this article is to develop optimal methods for plastic closure of septum perforations in children based on a retrospective analysis of results of surgical treatment.

MATERIALS AND METHODS: Plastic surgery closure of septum perforations using a newly developed method is described for 14 patients aged 6-15 years.

DISCUSSION: An analysis of the etiological factors affecting disease development is carried out, and the authors' technique of closing the subtotal perforations is described in detail, adapted for the child's age, and for preoperative preparation and postoperative management. In all operated patients, the postoperative period proceeded smoothly, without complications associated with the operation. A follow-up observation for more than a year after surgery showed no recurrence of septal perforation in patients from both groups.

CONCLUSION: A careful analysis of the causes, clinical manifestations, localization and sizes of septum perforation in comparison with the anatomical dimensions of intranasal structures allows one to individually select the optimal surgical method.
\end{abstract}

Keywords: nasal septum, perforation of the nasal septum, plastic closure of nasal septum

Address for correspondence:

Hassan Diab

Clinical Research Centre for Otorhinolaryngology to the

Federal Medico-Biological Agency of the Russian Federation

30 Volokolamskoe shosse St

123182 Moscow

Russia

e-mail:hasandiab@mail.ru

Received: June 18, 2021

Accepted: June 27, 2021

\section{INTRODUCTION}

For the surgical closure of septal perforations, a number of methods have been proposed, but none of them guarantees complete success. Failures in the surgical treatment are caused by the absence of clear individual indications for choosing a particular method for repairing the defect, which depends on its etiology, size and location. 
The emergence of a stable perforation is facilitated by poor nutrition of the cartilage and the imbalance between the cartilaginous, epithelial, and connective tissues (1). When the septum is perforated, the laminar flow of inhaled air becomes turbulent (2), thus causing deterioration in the normal physiological state of the ciliary epithelium of the nasal mucosa. This in turn causes degenerative and trophic changes that lead to chronic perichondritis and further necrosis of the cartilage. As a result, the size of the perforation increases, saddle-shaped deformation of the nose is formed, and the clinical symptoms worsen. Thus, the pathophysiological circle closes, and only a surgical treatment of the defect of the septum can break it (3).

Technical difficulties and failures are also determined by the fact that it is extremely rare to close the perforation by simple mutual displacement and cross-linking of the flaps of mucoperichondrium. More often, a complete, layered restoration of the septum skeleton (2-6) is required. The most promising method is that of closing septum perforations with the use of the mucous membrane of the lower nasal turbinate on a feeding stem. The material thus obtained most optimally matches by its histological structure the mucosa of the nasal septum in children (7-10).

Limitations on the use in pediatric practice of traumatic open access, various implants to close the defect of the septum, as well as the small dimensions of the nasal cavity and the continuing growth of the facial skeleton require a carefully weighted choice of the surgical method in each specific clinical case. Currently there are only a few reports on the experience of plastic closure of perforations of the nasal septum in children $(6,11-13)$.

\section{AIM}

The aim of this article is to develop optimal methods for plastic closure of septum perforations in children based on a retrospective analysis of results of surgical treatment.

\section{MATERIALS AND METHODS}

The protocol of the study was approved by the Ethics Committee of the Scientific and Clinical Center of Otorhinolaryngology, Moscow. Prior to the op- erations, informed consent was obtained from parents of the children.

The study group included fourteen children aged 6-15 years who were operated for a subtotal septal perforation with a diameter of up to $15 \mathrm{~mm}$.

The reason for the perforation of the nasal septum in five patients was a foreign body: a disk battery having remained in the nasal cavity from 1.5 hours to 24 days; six patients had a nasal septum injury; two patients had cauterization of vessels of the Kiesselbach zone, and one patient had a surgical intervention (septoplasty with a one-stage endoscopic polysinusotomy). In case of suspected Wegener's granulomatosis, an analysis was carried out for antineutrophil cytoplasmic autoantibodies (ANCAs). Patients with perforations of systemic origin were denied surgical treatment due to a high risk of recurrence and a progressive increase in the size of the perforation.

The survey included a thorough analysis of the patient's anamnestic data and complaints, nasal cavity endoscopy with rod-lens optics, measurement of the size and location of the perforation, photo data, computed tomography of the paranasal sinuses of nasal cavity, and a biopsy of the mucosa and cartilage from the posterior margin of perforation. The histological picture of morphological changes after a chemical burn of the mucous membrane of the nasal septum with a disk battery corresponded to postne-

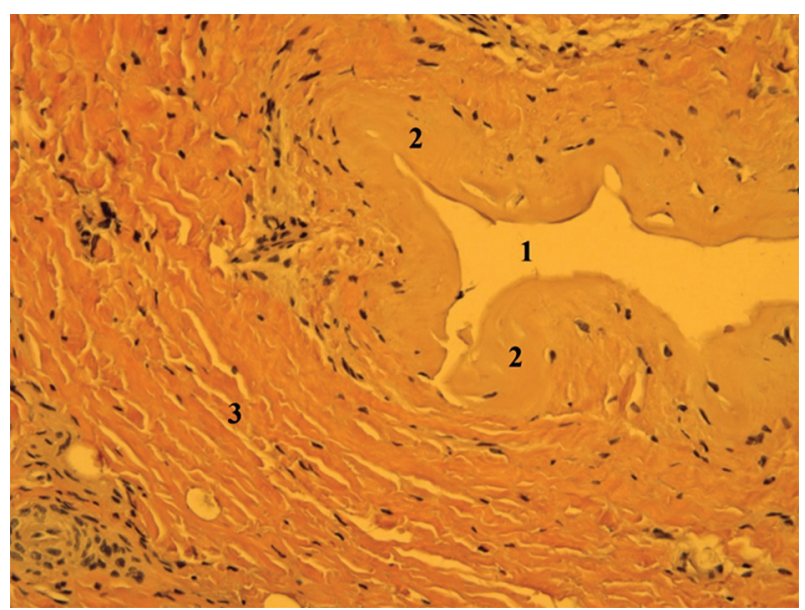

Fig. 1. A histological picture of morphological changes after chemical burn of the nasal septum mucosa by a disk battery: fields of hyalinized collagen fibers (1) with angiogenesis sites (2), hemosiderin deposition (3), minor diffuse lymphocytic infiltration; staining with hematoxylin-eosin (x200). 
Adnan Yunusov, Nikolai Daykhes, Hassan Diab et al.

crotic tissue changes. Fields of hyalinized collagen fibers with diffuse lymphocytic perivascular infiltration were determined in the preparations. Also, areas of angiogenesis and hemosiderin deposition were noted, which indicated a previously existing hemorrhage (Fig. 1).

As a preoperative treatment, the nasal cavity of all patients was washed and moistened by spraying with isotonic solutions based on sea salt, peach oil or oil solutions of vitamins A and E, instilled or applied on tampons; ointments with dexpanthenol were also used. Applications of methyluracil ointment were prescribed to patients with a large number of bloody crusts. Conservative treatment resulted in alleviation of the symptoms to some extent, but they did not disappear completely in any of the patients, which did not allow us or the parents to refuse surgical treatment.

Patients were divided into two groups. In the first group of 8 patients, a two-stage method of plastic closure of perforations with a flap from the inferior nasal concha on the feeding stem was employed (13). Under intubation anesthesia after hydro-preparation, the pathologically altered mucosa at the edges

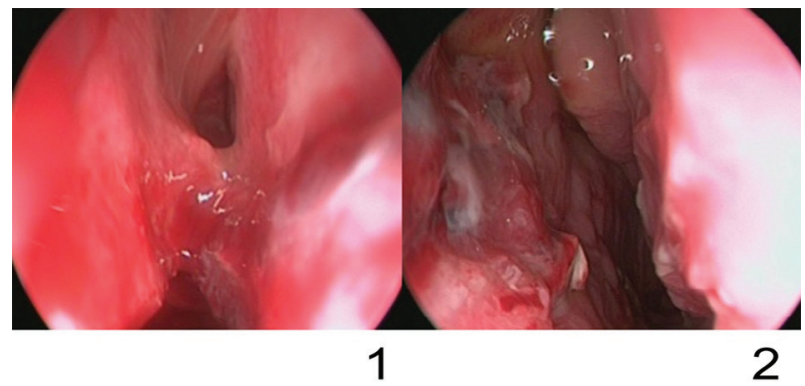

Fig. 2. An endoscopic picture after the first stage of closing the perforation of the nasal septum with a flap on a feeding stem: right view (1) and left view (2). of the perforation was excised circularly. Under the endoscopic control, the lower edge of the inferior nasal concha was cut off, forming a donor flap on the front feeding leg, including the mucous membrane and some of the submucosa layer, the thickness of which was cut in the middle to flatten and increase the area of the flap. The flap was mobilized anteriorly and sutured with circular absorbable sutures into the region of the nasal septum defect (Fig. 2).

On the lower wound surface of the donor nasal concha, under endoscopic control, thorough hemostasis was performed using various radio frequency nozzles in the coagulation regime. The operation was terminated by inserting elastic latex tampons with $5 \%$ synthomycine liniment into both halves of the nose for one day.

After the operation, cleaning of the nasal cavity was performed daily, followed by endoscopic monitoring, stamping the wound mucoperichondrium surfaces with Aecol, and parenteral antibiotics were administered.

The next stage of surgical treatment involved cutting off the feeding leg with radio-frequency destruction of the excess protruding tissue of the donor flap to restore full respiratory function on the donor half of the nose. This stage was not earlier than 3 months after the previous one (Fig. 3).

After the operation, to prevent the formation of synechia, the cavity of the donor half of the nose was daily swabbed with elastic latex tampons soaked with Aecol for 5-7 days. Complete epithelialization of the wound surfaces of the mucosa occurred 3 weeks later after the operation.

In the second group of 6 patients we employed a method of plastic closure of the septal perforation

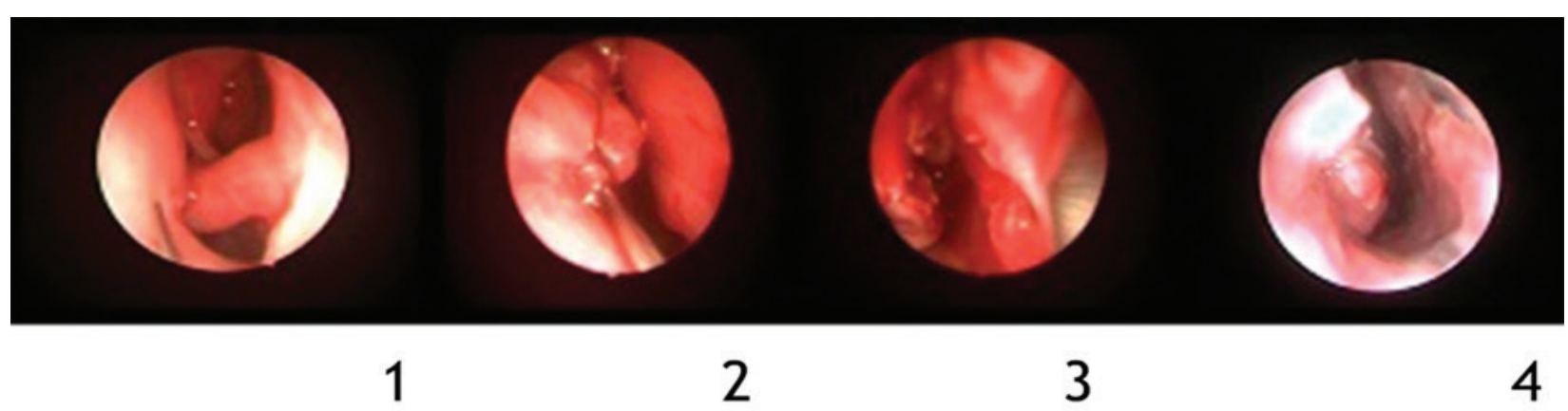

Fig. 3. An endoscopic picture of the second stage of closing the perforation of the nasal septum with a flap on a feeding stem: feeding stem (1), cutting off the feeding stem (2, 3), after the removal of latex tampons (4). 
with free transplantation of auto-tissue of the inferior turbinate, which was developed and patented by us (priority certificate of Patent of the Russian Federation of 11.11.2016, incoming number 071217, registration number 2016144413). The technique was as follows: under endotracheal anesthesia after circular removal of mucoperichondrium and mucoperiosteum throughout the quadrangular cartilage, under endoscopic control, a mucoperichondriosteum flap was cut out on the one side, starting from the highest part of columella, reaching the bottom of the nasal cavity, including the inner surface of the inferior turbinate. Next, the remainder of the cartilage was completely isolated, modeled and reimplanted with chondroinversion and moving its perforation site to the vomer. From this point of access, deformation of the premaxilla ridge was corrected in some cases, if indicated (Fig. 4).

The defect of the mucous membrane in the perforation region was covered on one side with the mobilized flap of the mucoperichondrium on the front and back feeding stems, with counter flaps sewing them with each other. On the opposite side, the defect was covered with a free flap of the mucous membrane of the inferior nasal concha cut from its middle and rear parts. The flap was placed under the mucoperichondrium leaflets in the region of the anterior part of the nose. Thus, the proximal edge of the flap was covered on its both sides, and the reimplanted
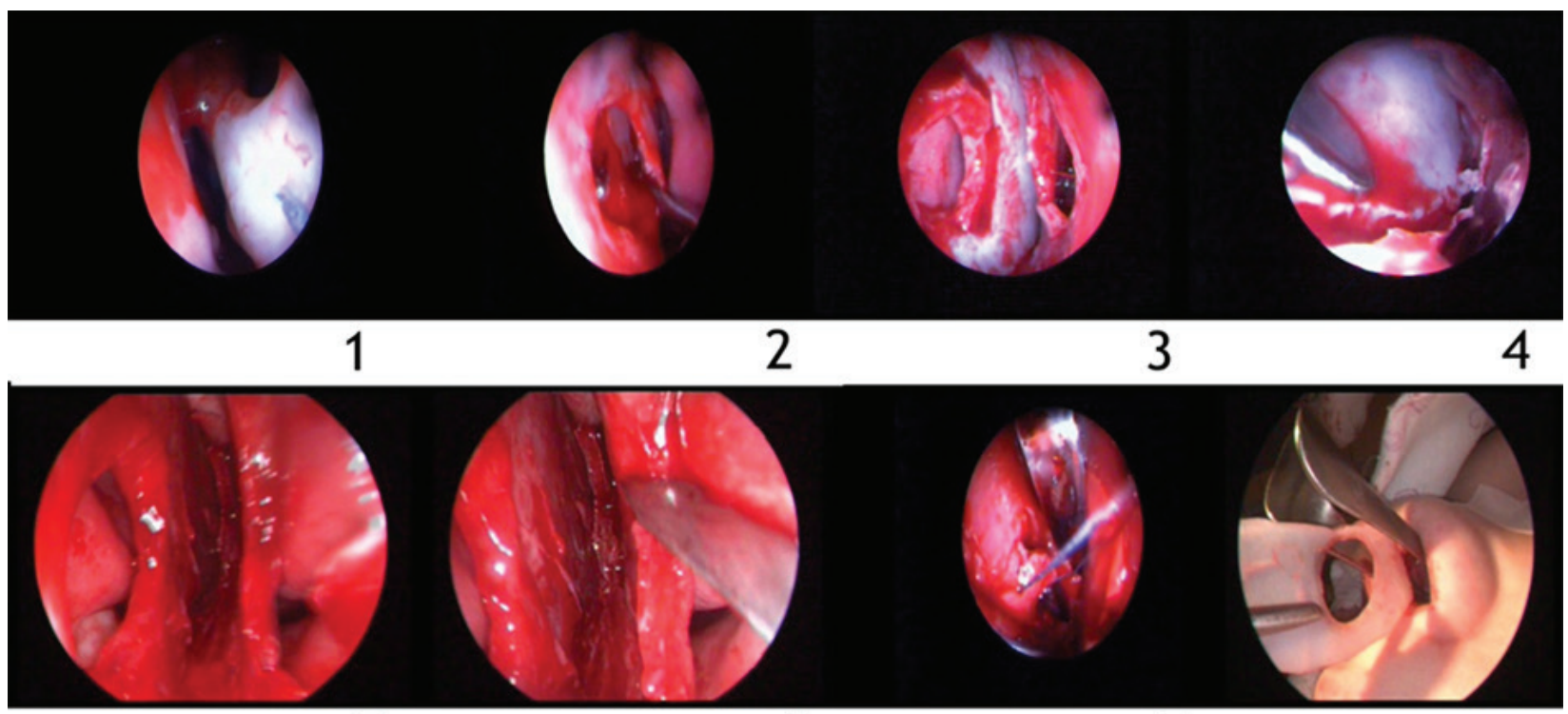

$2 \quad 3$

5

6
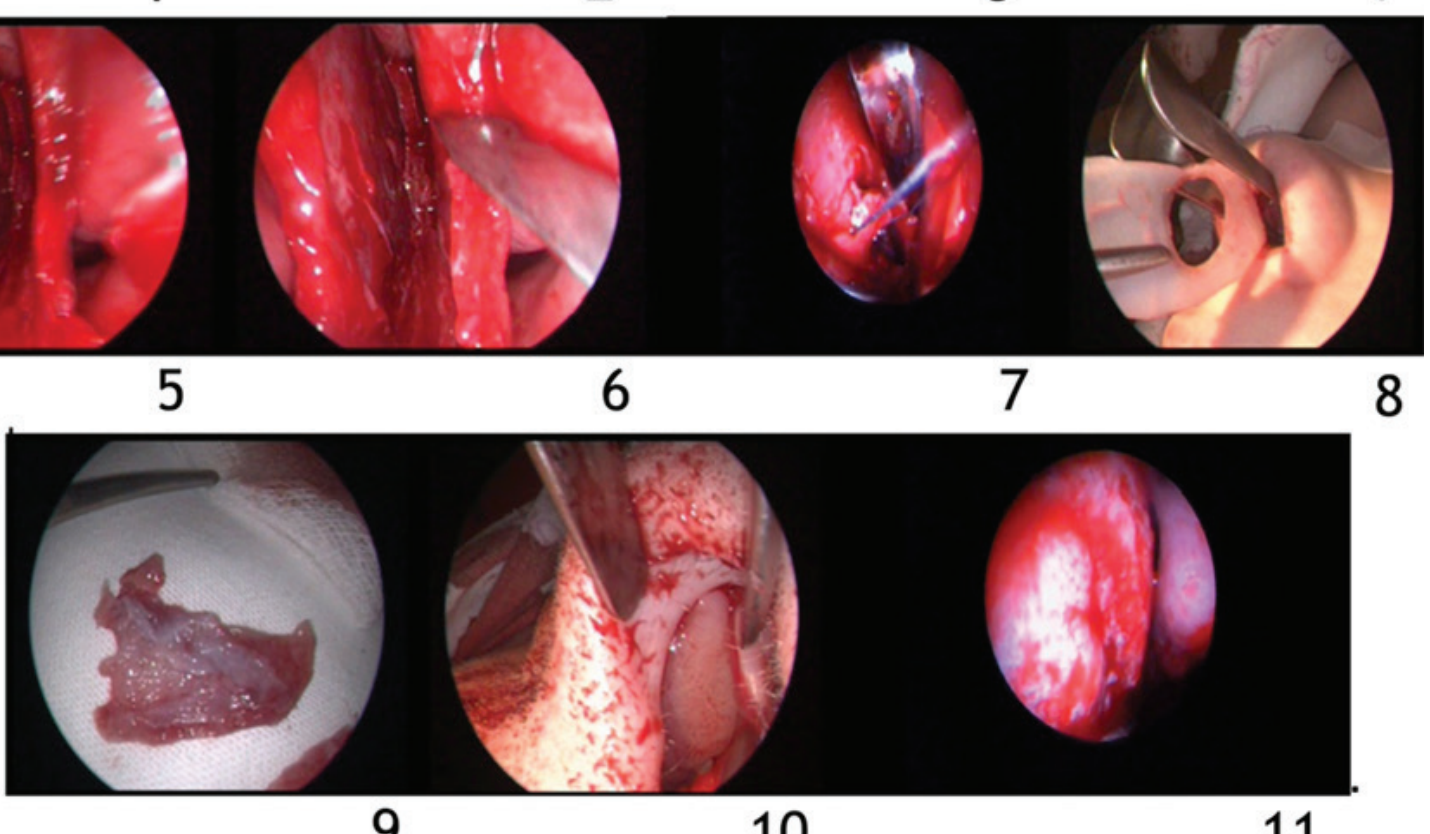

\section{9}

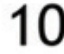

11

Fig. 4. An endoscopic picture of closing a perforation of the nasal septum with a free flap: hydropreparation of the mucosa (1), and dissection of mucoperichondrium and mucoperiosteum (2,3), mobilization of the quadrangular cartilage (4), separation of the quadrangular cartilage from the premaxilla (5), refreshment of perforation edges (6), stapling of mucosal flaps on one side (7) reimplantation of the cartilage by chondroinversion (8), the flap of the inferior turbinate (9), laying the flap between the sheets of mucoperichondrium and mucoperiosteum (10), the defect site is completely covered by the flap (11). 
cartilage was completely closed with a flap, the distal edge of which was placed on the perpendicular plate and vomer, and covered with a mucoperiosteum flap removed earlier. The newly formed construct of the restored septum was fixed with latex elastic tampons impregnated with $10 \%$ liniment of synthomycin (Fig. 5). The postoperative management was similar to that in the first group. of which the patients and their parents should be warned.

In cases of plastic closure of septal perforation using free transplantation of auto-tissues from the inferior nasal concha (the second group of patients), despite the significant traumatic nature of the method, the regeneration in the area of transplantation did not differ in comparison with patients from the
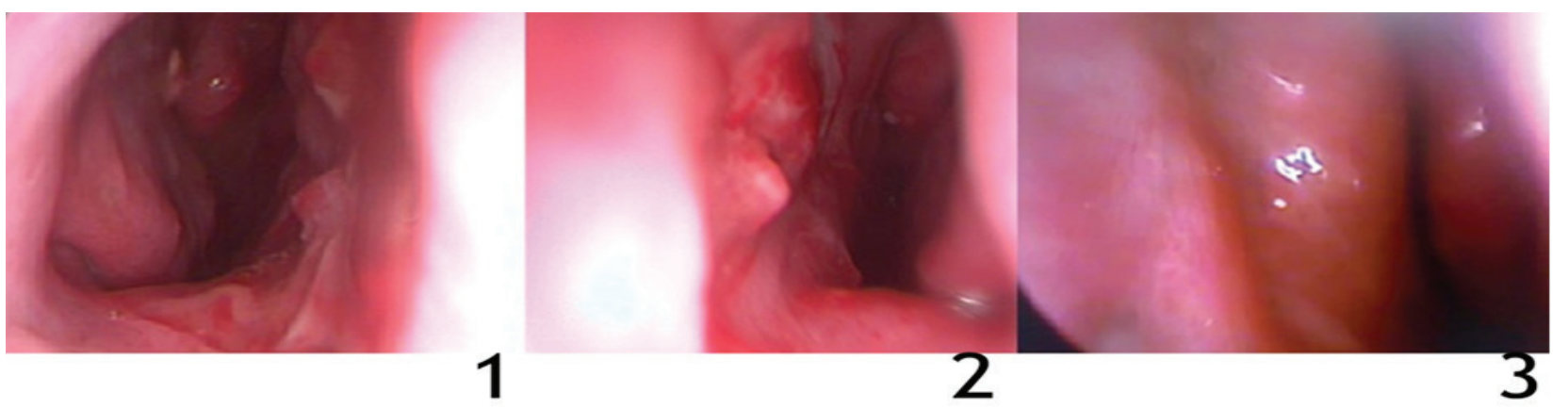

Fig. 5. An endoscopic picture after closure of the perforation of the nasal septum with the use of free transplantation of auto-tissues from the inferior nasal concha: right view (1) and left view (2), and left view 2 months after the operation

(3).

\section{DISCUSSION}

In all operated patients, the postoperative period proceeded smoothly, without complications associated with the operation. A follow-up observation for more than a year after surgery showed no recurrence of septal perforation in patients from both groups.

The size of the donor flap directly depends on the size of the inferior nasal concha. To close small perforations, we used only one-sided flaps, but theoretically, if there is a shortage of plastic material or a recurrence of perforation, we could successfully use a second similar donor flap of the inferior nasal concha from the opposite half of the nose.

It should be noted that an important nuance of using a two-stage method of plastic closure of perforations with a flap from the inferior nasal concha on the feeding stem (the first group of patients) is the fact that the healing of the wound surface from the opposite side of the donor flap proceeds by way of secondary closure and complete epithelialization occurs 3-4 weeks after the operation. This sometimes requires a later withdrawal of stitches (on $7^{\text {th }}-8^{\text {th }}$ day) as well as a longer local treatment and monitoring. Also, after the operation, there is a natural worsening of nasal breathing by the donor half of the nose first group. In about one month, a free mucosal graft in the area of the former perforation acquired pink shades and differed little from the surrounding mucosa of the septum. Probably, this is due to sufficient trophism when the edges of the transplanted free flap are under submucoperochondrial conditions. The important advantages of the developed method are: one-stage implementation; complete, layered restoration of the skeleton of the septum of the nose in the area of the defect; and the possibility of plastic closure of perforations greater than $15 \mathrm{~mm}$ in diameter.

\section{CONCLUSION}

A careful analysis of the causes, clinical manifestations, localization and sizes of septum perforation in comparison with the anatomical dimensions of intranasal structures allows one to individually select the optimal surgical method and, given a certain rhinosurgical experience, successfully perform the plastic closure in children. 


\section{Conflict of interests}

None to declare.

\section{Acknowledgements}

This work was supported by Scientific and Clinical Center of Otorhinolaryngology at the Federal Medical-Biological Agency of the Russian Federation.

\section{REFERENCES}

1. Apostolidi, KG, Epifanov SA, Krainik IV, Beksha IS, Krainik VI, Michailov AI. Plastic surgery for elimination of deformation and perforation of the nasal septum. Journal N I Pirogov Natl Med Surg Center. 2011;3:114-5. (in Russian).

2. Molokov KV. Perforation of the nasal septum - causes and treatments. MD thesis; 2007. (in Russian).

3. Meyer R. Nasal septal perforations must and can be closed. Anesthetic Plast Surg. 1994;18(4):345-55. doi: 10.1007/BF00451339.

4. Ustyanov YA. To the problem of the treatment of nasal septum perforation. Rus Rhinol. 1996; 65-72. (in Russian).

5. Lopatin AS, Ovchinnikova EV. Peculiarities of surgical treatment of perforations of the nasal septum. Vestn Otorinolar. 2012;2:13-7 (in Russian).

6. Yunusov AS, Rybalkin SV. A method of plastic closure of subtotal perforation of the nasal septum. RF Patent for invention no.2246910 of 27.02.2005. (in Russian).
7. Murakami CS, Kriet JD, Ierokomos AP. Nasal reconstruction using the inferior turbinate mucosal flap. Arch Facial Plast Surg. 1999;1(2):97-100. doi: 10.1001/archfaci.1.2.97.

8. Friedman M, Ibrahim H, Ramakrishnan V. Inferior turbinate flap for repair of nasal septal perforation. Laryngoscope. 2003;133(8):1425-8. doi: 10.1097/00005537-200308000-00031.

9. Kilty SJ, Brownrigg PJ, Safar A. Nasal septal perforation repair using an inferior turbinate flap. J Otolaryngol. 2007; 36(1):38-42. doi: 10.2310/7070.2005.0168.

10. Tastan E, Sozen T. Oblique split technique in septal reconstruction. Facial Plast. Surg. 2013;29(6):48791. doi: 10.1055/s-0033-1360599.

11. Chua DY, Tan KH. Repair of nasal septal perforations using auricular conchal cartilage graft in children: report on three cases and literature review. J. Pediatr Otorhinolaryngol. 2006;70(7):1219-24. doi: 10.1016/j.ijporl.2005.12.021.

12. Rusetsky YY, Spiranskaya OA. Latysheva EN, Chernishenko IO, Machambetova EA. The experience of the plastic closure of perforation of the nasal septum in children and adolescents. Rus Rhinol. 2016;24:10-5. (in Russian).

13. Yunusov AS., Dayhes NA., Rybalkin SV. Reconstructive rhinosurgery in children. Moscow: TRIADA Publishers; 2016. pp. 110-6. (in Russian). 